\title{
A Dynamic Loading Test for Evaluating Permanent Deformation Resistance of Asphalt Mixtures
}

\author{
Xiaoguang Xie ${ }^{1, a}$, Long Wang ${ }^{2, b}$,Xiaorui Zhang ${ }^{3, c}$ \\ ${ }^{1}$ School of Transportation Science and Engineering Harbin Institute of Technology Harbin \\ 150090, China \\ ${ }^{2}$ School of Transportation Science and Engineering Harbin Institute of Technology Harbin \\ 150090, China \\ ${ }^{3}$ School of Transportation Science and Engineering Harbin Institute of Technology Harbin \\ 150090, China
}

axxg75@126.com , bhitlongwang@sina.com , chagongdadaolu@163.com

\begin{abstract}
Keywords: asphalt mixtures; deformation resistance; dynamic loading; permanent deformation
\end{abstract}
\begin{abstract}
Recent research shows that there is a serious need for accurate methods to evaluate the deformation resistance of asphalt mixtures at high temperatures, especially for stone-skeleton mixtures. To reflect the interlocking angle between coarse aggregates, confining pressure should be considered during testing. The dynamic loading test has been developed for applying different repetitive loads to a limited area of the specimen. This test can measure permanent deformation indices under different loads to evaluate the deformation resistance of asphalt mixtures at high temperature. The dynamic loading test is conducted on samples with different compaction rates and gradations. Further, the rutting resistance of two types of gradation is compared with a wheel tracking test and a dynamic loading test. The test results indicate that the dynamic loading test can apparently distinguish the influence of gradations on rutting resistance. It is also derived from this test that dense-graded mixtures have higher permanent deformation susceptibility than coarsegraded mixtures, which is consistent with experience in the field and validated by experiments. This test can reflect the entire permanent deformation and separate the compactive deformation and shear flow deformation. Furthermore, this test can simulate heavy and repeated loads to fully exhibit the deformation resistance of asphalt mixtures, especially for coarse-graded mixtures. However, this test is limited by the effects of rigid confinement, which can be improved by changing the confining pressure.
\end{abstract}

\section{Introduction}

At present, the "working environment" of roads are becoming more complex and severe. High traffic volumes and tire pressures combined with high ambient temperature accelerates the deterioration of asphalt pavement. Now one of the most common forms of failure of asphalt pavement is rutting [1]. For semi-rigid base asphalt pavement, rutting is primarily derived from the permanent deformation of the asphalt concrete layer at high temperatures. The gradation of hot-mix asphalts (HMA) should be accurately designed to improve rutting resistance. Literature research shows that $60 \%$ rutting resistance of asphalt mixtures is attributed to the interlocking forces provided by coarse particles, the other $40 \%$ coming from the cohesion of asphalt binder. In China, more and more asphalt-treated base (ATB) and asphalt-treated permeable base (ATPB) are applied to reduce the reflective cracking and moisture damage arising from the used semi-rigid base [2]. Therefore, it is essential to accurately evaluate the deformation resistance of stone-skeleton asphalt mixtures.

There are many methods to judge rutting resistance of asphalt mixtures including wheel tracking (WT), static creep, dynamic creep, indirect tensile strength (ITS) tests and an accelerated loading facility (ALF) test. ITS test is developed for elastic base and does not accurately estimate the rutting resistance of a mixture. Wheel-tracking tests are not quantified to be well correlated with rutting in the field under variable traffic loading and environmental conditions. However, it can be 
used to rank mixtures according to rut potential. Wheel-tracking tests generate two rutting parameters (rutting depth and dynamic stability). Dynamic stability has limitation and sometimes conflicts with rutting depth, for it considers only the rutting depth at $45 \mathrm{~min}$ and $60 \mathrm{~min}$, not the whole depth [3]. Experiences have shown that stone-skeleton mixtures, such as SMA and ATB, cannot be properly evaluated by means of conventional tests, such as the unconfined uniaxial static or dynamic creep tests [4]. Other studies for rutting evaluation use a creep loading or repeated loading in uniaxial or triaxial modes, however, these test procedures are relatively complex and are not widely used in field laboratories [5,6].

The existing experimental tests have limitations and lack rationalities to evaluate the deformation resistance of HMA, especially for stone-skeleton mixtures. It is essential to select an accurate test to evaluate the deformation properties of asphalt mixtures. The shear strength of stone-skeleton mixture is mainly derived from interlocking angle, $\varphi$ used in the shear strength equation

( $\tau=c+\sigma \operatorname{tg} \varphi$ ). To reflect the interlocking angle, $\varphi$ confining pressure, $\sigma$ should be considered. In general, rutting occurs mainly on wheel paths in the roads, the rest of the pavement section remaining almost intact. The spot under the wheel suffers stress and the immediate surrounding body acts as a restraining barrier for shear movement when the material underneath the tire begins to deform. According to this hypothesis, instead of applying load to the whole cross-sectional area of a specimen, a test method is developed to apply a dynamic load to a limited area with confining pressure. This test can reflect the deformation resistance of asphalt mixtures, especially for stoneskeleton mixtures at high temperatures, called as dynamic loading test.

\section{Development of Dynamic Loading Test}

Repetitive load tests apply an axial dynamic stress on a specimen with a sinusoidal loading pattern, close to the actual field loading conditions. Repetitive load tests are also thought to be more accurate than static testing to evaluate the characteristics of asphalt mixtures. The illustration above is the main reason to develop dynamic loading test in this research. Fig. 1 illustrates a periodic load applied to a specimen in a limited area (referring to CBR test). The applied load is haversine in shape and frequency is 0.1 hertz. Each level load increases by $10 \mathrm{kN}$ and 100 repetitions. The deformation curves under different load level are plotted through a sensor and displacement collecting system, as shown in Fig. 2. The elastic and plastic deformation can be directly derived from the deformation curve. The other deformation indices can be calculated, such as accumulative deformation, resilient and plastic modulus (equation 1).

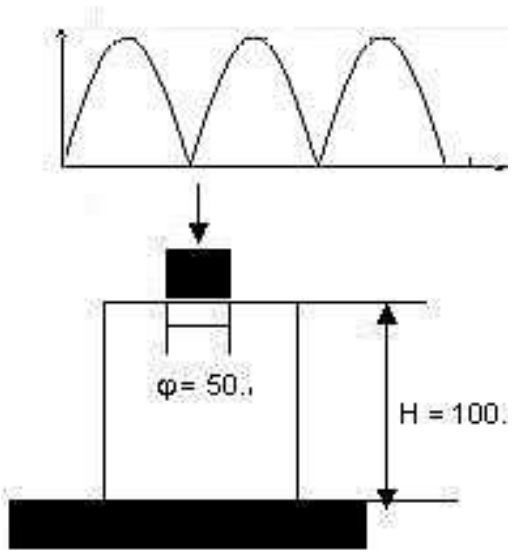

Fig. 1 Dynamic loading test (mm)

$$
E=\frac{\pi D}{4} \times \frac{p}{l}\left(1-\mu_{0}{ }^{2}\right)
$$

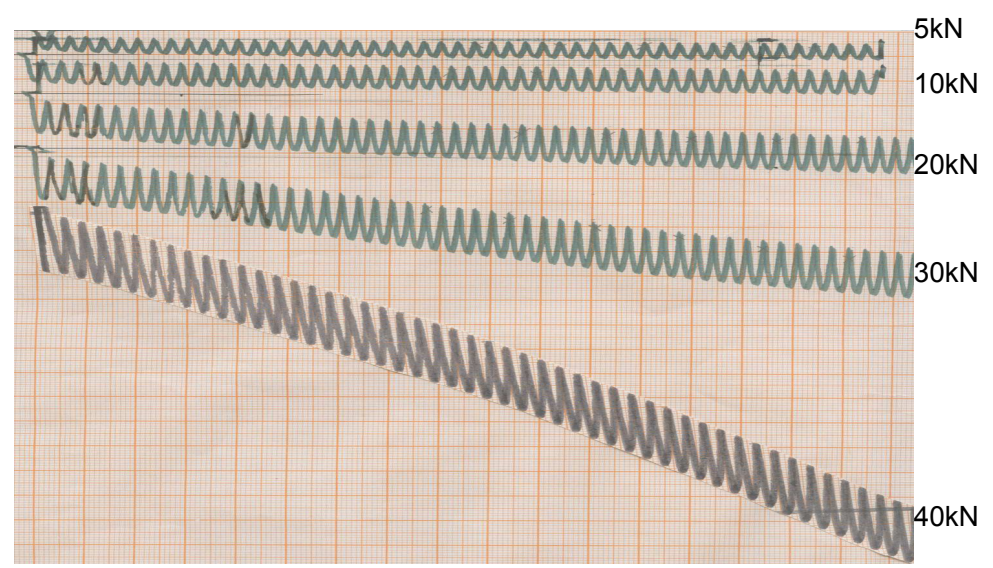

Fig. 2 Dynamic deformation curve of asphalt mixture at various loading 
Where:

$E$-resilient or plastic modulus ( $\mathrm{MPa})$;

$\mu_{0}$ - poisson ratio $(0.35)$;

$D$ - the diameter of loading head $(50 \mathrm{~mm})$;

$p$-loading stress ( $\mathrm{MPa})$;

$l$-elastic or plastic deformation $(\mathrm{mm})$;

\section{Characteristics of the Test}

The purpose of developing the new test method is to create a dynamic load-induced spot deformation similar to the one created by moving wheel. The dynamic loading test has a head $(\phi=$ $50 \mathrm{~mm})$ on the specimen $(\phi=150 \mathrm{~mm})$ to simulate a variable wheel which has a circular tire imprint. Considering confining pressure, the dynamic loading test can evaluate interlock function between coarse aggregates under different loading levels, which is consistent with the actual stress state of asphalt pavement.

Permanent deformation in pavements has long been recognized to include two different modes according to Huang and Gokhale. The first mode is known as compactive deformation (consolidation of layers) and the second mode is plastic deformation (asphalt shear flow). The test system applies a dynamic load step-by-step from $5 \mathrm{kN}$ to $40 \mathrm{kN}$, aiming to simulate the accumulative process of permanent deformation to attain different deformation stages. When the applying load is minor, deformation is known as compactive stage (the first mode). When the applying load increases, permanent deformation will increase consequently, the variable amplitude is not large, and deformation is known as accumulative stage. When the applying load increases further, the slope of deformation becomes larger sharply, which means the specimen failed, this deformation is known as shear flow. Fig. 2 shows the trend of deformation of an asphalt mixture.

The elastic and plastic deformation under different loading are separated from the test data. Further, the resilient modulus and permanent deformation can be calculated. The stress state is explicit in the uniaxial or triaxial creep tests and the objective is the cell, which represents the stress state of one point in the pavement. While the stress state of homogenate is nonuniform along the longitudinal and transverse distribution, the dynamic loading test reflects the entire homogeneous structure.

Loading Mode. The dynamic test with haversine loading cycles is repeated continually without any interval period. The repeated loadings are applied by a MTS machine. The maximum amplitudes are $5 \mathrm{kN}, 10 \mathrm{kN}, 20 \mathrm{kN}, 30 \mathrm{kN}$, and $40 \mathrm{kN}(2.55 \mathrm{MPa}, 5.09 \mathrm{MPa}, 10.19 \mathrm{MPa}, 15.29$ $\mathrm{MPa}$ and $20.38 \mathrm{MPa}$, respectively). The frequency is 0.1 hertz and cycle number is 100 each time.

Specimen Dimension. The stress distribution of the specimens is qualitatively analyzed using the finite element program in different heights and diameters. When specimen height is less than $100 \mathrm{~mm}$, the position of the maximum normal stress is nearly half of the height. When specimen height is $150 \mathrm{~mm}$, the position of the maximum normal stress value is $2 / 3$ at $100 \mathrm{~mm}$ height. Therefore, the specimen dimension values selected are $\varphi=150 \mathrm{~mm}$ and $\mathrm{H}=100 \mathrm{~mm}$.

Experiment Temperature. The test temperature of evaluating deformation resistance of HMA at high temperature is, in general, between $40^{\circ} \mathrm{C}$ and $60^{\circ} \mathrm{C}$. Through the summary of the literature, $45^{\circ} \mathrm{C}$ is selected for the asphalt mixture to evaluate permanent deformation resistance under high temperature.

\section{Experimental Research}

Materials. To prove the test susceptibility, five aggregate gradations are chosen, in which framework-void structure of asphalt mixtures are dominant, the other are dense-graded asphalt mixtures. Limestone aggregate is sampled from the Jilin rock quarry in China, and the 80-100 penetration asphalt cement comes from Panjin northern asphalt refinery. All materials and gradations demand Technical Specifications for Construction of Highway Asphalt Pavements in 
China. Table 1 shows five used gradations. The gradation name respectively represents coarse aggregate, skeleton number-Taibo, an exponent $n$ value of fine aggregate-filling coefficient. Every specimen should be brought to bear on different load level (2.55 MPa, 5.09 MPa, 10.19 MPa, 15.29 $\mathrm{MPa}$ and $20.38 \mathrm{MPa}$ ).

Table 1 Five Gradations in the Experiment Tests

\begin{tabular}{c|c|c|c|c|c|c|c|c|c|c|c|c}
\hline Gradation & AirVoid & \multicolumn{10}{|c}{ Seize Size $(\mathrm{mm})$ Percentage Passing (\%) } \\
\cline { 3 - 12 } Name & $(\%)$ & 19.0 & 16.0 & 13.2 & 9.5 & 4.75 & 2.36 & 1.18 & 0.6 & 0.3 & 0.15 & 0.075 \\
\hline $4-0.65-0.5$ & 24.0 & 100 & 88.3 & 70.4 & 33.7 & 22.0 & 14.0 & 8.9 & 5.7 & 3.7 & 2.3 & 1.5 \\
$4-0.65-0.7$ & 18.5 & 100 & 89.2 & 72.6 & 38.8 & 28.0 & 17.8 & 11.3 & 7.3 & 4.6 & 3.0 & 1.9 \\
$4-0.65-0.9$ & 14.5 & 100 & 90.1 & 74.9 & 43.9 & 34.0 & 21.6 & 13.8 & 8.9 & 5.6 & 3.6 & 2.3 \\
$4-\mathrm{N}-0.9$ & 2.2 & 100 & 90.0 & 74.9 & 43.9 & 34.0 & 32.0 & 28.0 & 23.0 & 17.0 & 12.0 & 8.0 \\
$1-\mathrm{N}-0.9$ & 1.5 & 100 & 69.0 & 53.8 & 43.9 & 34.0 & 32.0 & 28.0 & 23.0 & 17.0 & 12.0 & 8.0 \\
\hline
\end{tabular}

Aggregate Gradation Influence. Five gradations including 3 coarse-graded mixtures and 2 dense-graded mixtures (see Table 1) are fully compacted by the optimum compaction rate. Accordingly, deformation and modulus values are shown in Fig. 3 to Fig. 6.

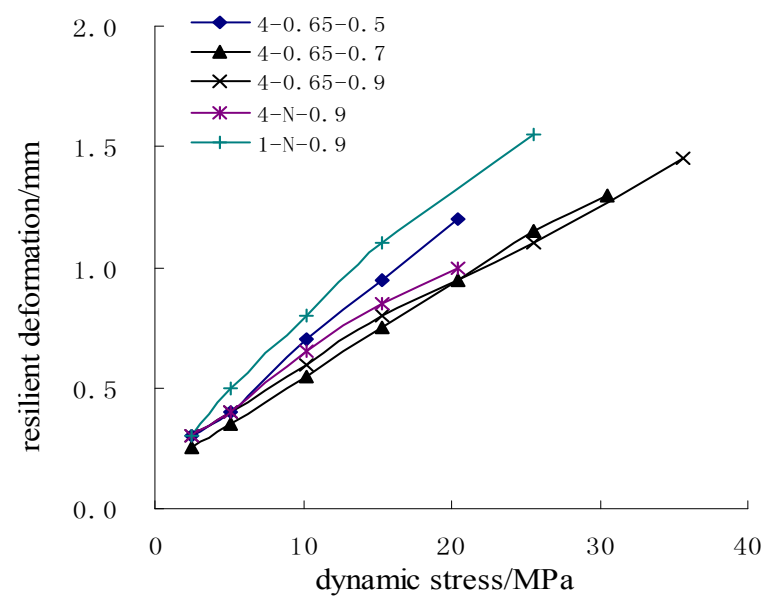

Fig. 3 Dynamic stress and resilient deformation of different gradations.

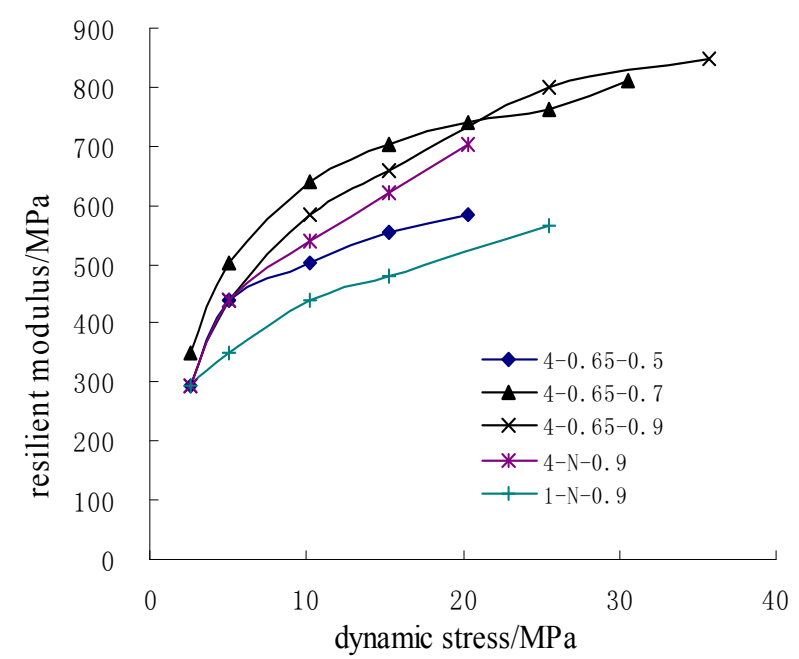

Fig. 5 Dynamic stress and resilient modulus of different gradations.

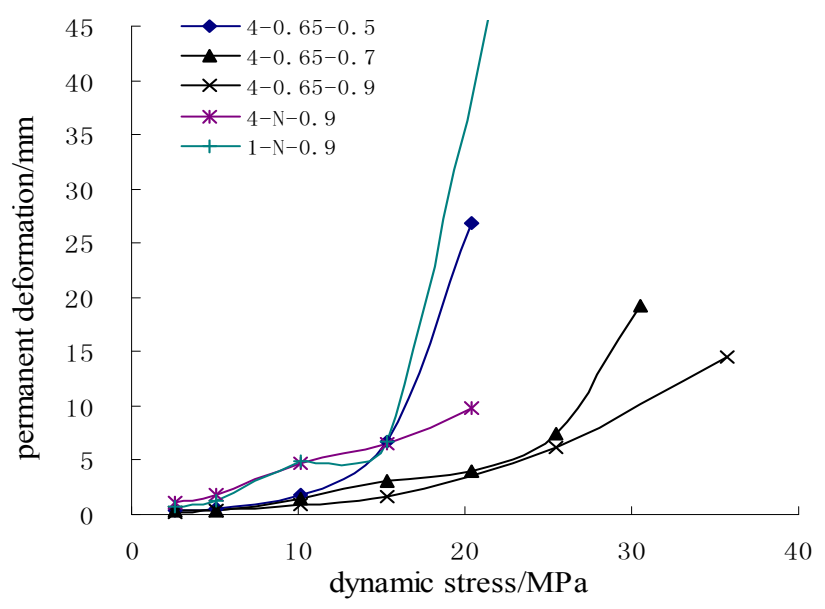

Fig. 4 Dynamic stress and permanent deformation of different gradations.

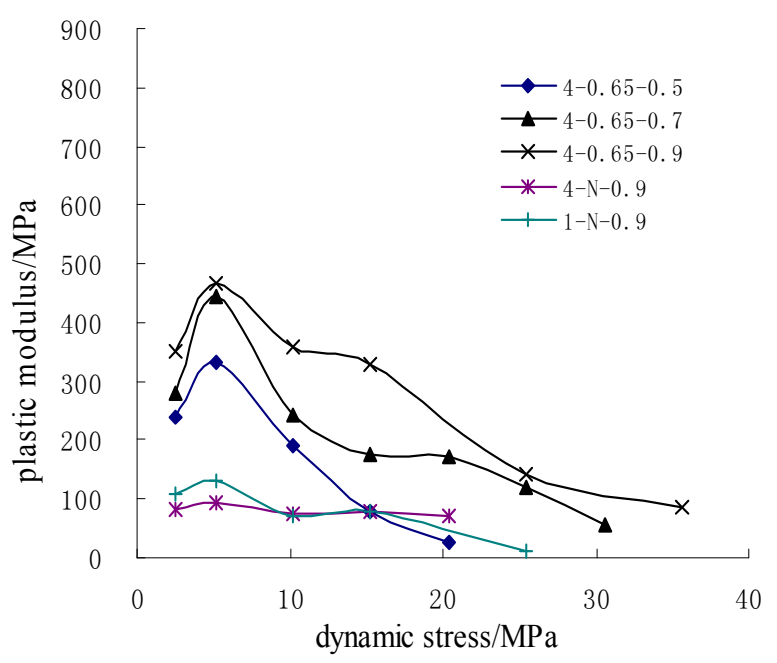

Fig. 6 Dynamic stress and plastic modulus of different gradations. 
It can be clearly observed for both trends that as dynamic stress increases, resilient deformation and resilient modulus of different gradations are similar. There is a discrepancy in resilient deformation of 0 to $0.5 \mathrm{~mm}$ at the same dynamic stress (Fig. 3), while resilient modulus (Fig. 5) is 0 to $200 \mathrm{MPa}$. However, permanent deformation is quite different for different gradations. A discrepancy in permanent deformation is about 0 to $20 \mathrm{~mm}$ at the same dynamic stress (Fig. 4), while plastic modulus (Fig. 6) is 150 to $400 \mathrm{MPa}$. In Fig. 4, all five gradation mixtures are compacted efficiently, so permanent deformations under minor dynamic stress (less than $10 \mathrm{MPa}$ ) are nearly equal, which shows that asphalt mixtures compacted fully have little compactive deformation, regardless of any kind of aggregate gradation. While as dynamic stress increases (more than $25 \mathrm{MPa}$ ), the permanent deformations of different gradations are mainly attributed to asphalt shear flow, the value revealing significant distinctions of different gradations. Thus, it can be concluded that the discrepancy of deformation resistance of different gradation at high temperature can be measured through the dynamic loading test.

Fig. 6 shows that the plastic modulus reaches a maximum value at $5.09 \mathrm{MPa}$. As the dynamic stress increases, plastic modulus decreases. This indicates that when dynamic stress is no more than 5.09 $\mathrm{MPa}$, deformation increases slowly and exhibits increasing modulus. As dynamic stress becomes more than $5.09 \mathrm{MPa}$, permanent deformation increases quickly and exhibits decreasing plastic modulus. It can be concluded that $5.09 \mathrm{MPa}$ is a threshold for permanent deformation of asphalt mixtures, that is, compactive deformation in no more than 5.09 $\mathrm{MPa}$ and shear flow deformation in more than 5.09 $\mathrm{MPa}$. It can be seen that this test reflects the entire deformation of asphalt mixture at high temperature.

Through analysis of coarse skeleton and filling coefficient of five asphalt mixtures and their influence on performance, it can be concluded that the coarse skeleton 4 bears heavier loading than coarse skeleton 1, which is in agreement with the research results of graded crush stone[7,8]. Fig. 4 and Fig. 5 show that an asphalt mixture with a filling coefficient of 0.9 is the optimum at the same coarse skeleton 4. Fig. 7 also displays the comparison results of the permanent deformation of dense-graded (4-N-0.9 and 1-N-0.9) and coarse-graded asphalt mixtures (such as 4-0.65-0.9) at $45^{\circ} \mathrm{C}$ temperature under different stress levels. Permanent deformation resistance of coarse-graded samples is more than dense-graded samples especially at heavy loads. This conclusion is validated by experience and field work, which also proves that dynamic loading test can reflect the deformation resistance of different types of gradations.

Contrast Analysis. Two aggregate gradations (4-0.65-0.9, 4-N-0.9) are used for preparing the specimen for the WT test and dynamic loading test. The curve in Fig. 7 shows accumulative permanent deformation increases along the time under different tire pressures. In general, with the tire pressure (from $0.7 \mathrm{MPa}$ to $1.0 \mathrm{MPa}$ ) increases, vertical permanent deformation and the slope of asphalt mixtures increase too. When tire pressure increases to $1.15 \mathrm{MPa}$, the deformation slope is sharp before $20 \mathrm{~min}$, then it slows. This phenomenon reveals two things, one is that dynamic stability derived from the deformation slope between $45 \mathrm{~min}$. and $60 \mathrm{~min}$. reflects the rutting resistance of asphalt mixtures, although not the whole permanent deformation; and secondly, such a phenomenon is possible that the dynamic stability of different asphalt mixtures are the same, while permanent deformations may be quite different. For instance, the deformation slopes of two gradations are similar, but the permanent deformation becomes different at $60 \mathrm{~min}$. WT can exhibit the dynamic stability value under minor load $(0.7 \mathrm{MPa})$, but it produces an error and leads to unreliable statistics in lieu of dimension effect when applying loads are heavy. Fig. 7 shows that the deformation slope of two gradations between $45 \mathrm{~min}$. and $60 \mathrm{~min}$. are the same under $1.15 \mathrm{MPa}$, but quite different in accumulative permanent deformation. Fig. 8 clearly presents that as dynamic stress increases, accumulative permanent deformation increases with different slopes for two gradations. The permanent deformation under different loads is able to be directly measured through the dynamic loading test, which significantly proves that the dynamic loading test effectively reflects the permanent deformation under different loads. 


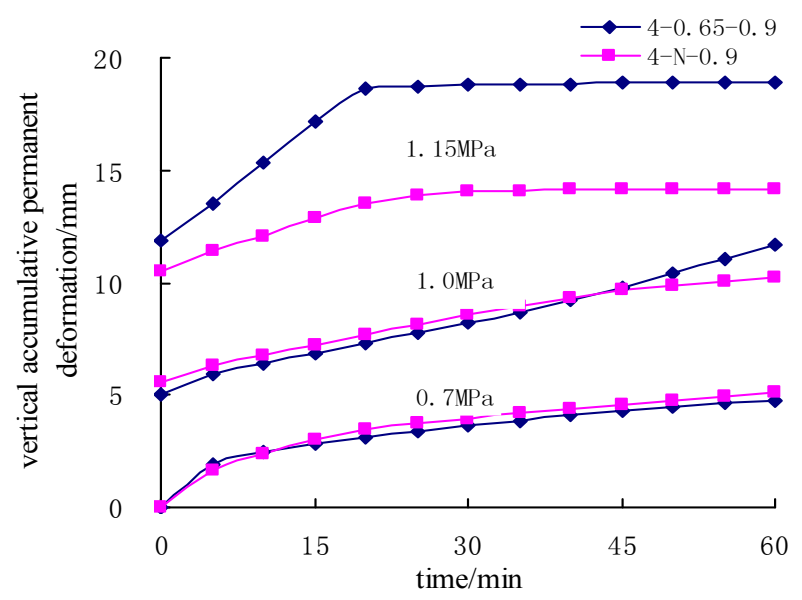

Fig. 7 Time and accumulative permanent deformation under different tire pressures.

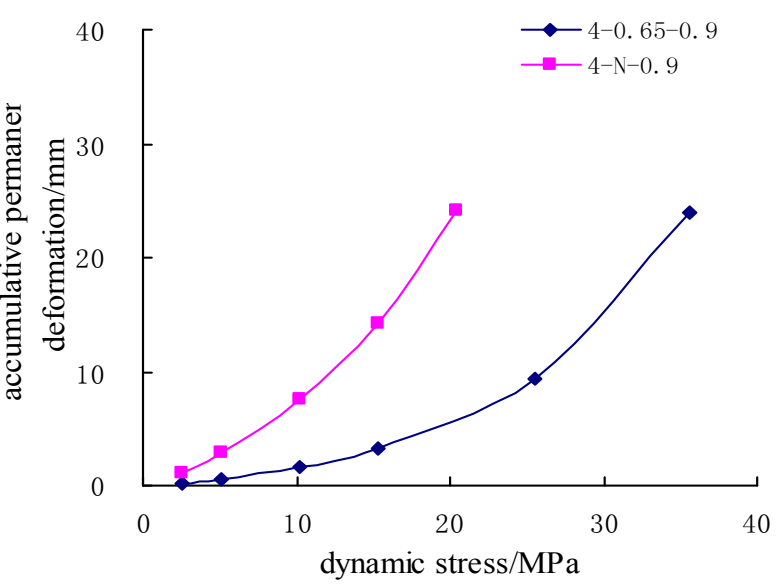

Fig. 8 Dynamic stress and accumulative permanent deformation.

\section{Conclusions}

For coarse skeleton asphalt mixtures, the shear strength is mainly derived from the interlocking forces provided by coarse aggregates. It is accurate to evaluate the deformation resistance of HMA considering the existence of confining pressure. The dynamic loading test is developed for applying different repetitive loads to a limited area of the specimen and utilizing mold confinement to evaluate the deformation resistance of asphalt mixtures at high temperatures.

This test can reflect the entire permanent deformation of asphalt mixtures at high temperature and separate the compactive deformation and shear flow deformation. Furthermore, the test can simulate different loads, especially heavy and repeated loads, which could fully exhibit the deformation resistance of asphalt mixtures, especially for coarse-graded mixtures.

The test is limited by the rigid confinement, which could be improved by changing the confinement.

\section{References}

[1] Tayfur, S., H. Ozen, and A. Aksoy. Investigation of Rutting Performance of Asphalt Mixtures Containing Polymer Modifiers. Construction and Building Materials, Vol. 21, 2006, pp. 328-337.

[2] Technical Specifications for Construction of Highway Asphalt Pavements in China. JTG, F40, 2004.

[3] Huihong, H. Assessment on Property of Wheel Tracks in Asphalt Mixtures. Master's Thesis. Harbin Institute of Technology, Harbin, P.R. China, 2000.

[4] Visser, A. T., F. Long, A. Verhaeghe, and A. Taute. Provisional Validation of the New South African Hot-mix Asphalt Design Method (Mix Design-1:7-6). Ninth International Conference on Asphalt Pavements. Copenhagen, Denmark, August 2002, p. 36-47.

[5] Krutz, N. C., R. Siddharthan, and M. Stroup-Gardiner. Investigation of Rutting Potential Using Static Creep Testing on Polymer-Modified Asphalt Concrete Mixture. In Transportation Research Record (TRR): Journal of the Transportation Research Board, No. 1317, Transportation Research Board (TRB) of the National Academies, Washington, D.C., 1991, pp. 100-118.

[6] Little, D. N. Performance Assessment of Binder of Binder-Rich Polyethylene-Modified Asphalt Concrete Mixtures (Novophalt). In TRR: Journal of the TRB, No. 1317, TRB of the National Academies, Washington, D.C., 1991, pp. 1-9.

[7] El Hussein, H. M., and Z. Yue. Criteria for Evaluation of Rut Potential Based on Repetitive Uniaxial Compression Test. In TRR: Journal of the TRB, No. 1454, TRB of the National Academies, Washington, D.C., 1994, pp. 74-81.

[8] Brown, S. F., and J. M. Gibb. Validation of Experiments for Permanent Deformation Testing of Bituminous Mixtures. Journal of Asphalt Paving Technology, Vol. 65, 1996, pp. 255-299. 